\title{
Stakeholder Values and Preferences in Lower Limb Amputation for No-Option Chronic Limb Threatening Ischemia
}

\author{
Jelle A Nieuwstraten (D) \\ Louk P van Doorn (D) \\ Winifred A Gebhardt ${ }^{2}$ \\ Jaap F Hamming'
}

'Department of Vascular Surgery, Leiden University Medical Center, Leiden, the Netherlands; ${ }^{2}$ Health, Medical and Neuropsychology Unit, Institute of Psychology, Leiden University, Leiden, the Netherlands
Correspondence: Jelle A Nieuwstraten Department of Vascular Surgery, Leiden University Medical Center, Postzone K6R, Postbus 9600, Leiden, RC, 2300, the Netherlands

Tel +31 618849873

Email Jellenieuwstraten@gmail.com
Purpose: This study focusses on identifying values and preferences of patients, caregivers and healthcare professionals who have dealt with lower limb amputation for no-option chronic limb threatening ischemia. No-option chronic limb threatening ischemia is defined as limb ischemia for which no treatment options exist and where lower limb amputation is necessary in the short term. The values and preferences identified in this study can help improve decision-making processes.

Patients and Methods: This was a qualitative study, using semi-structured interviews to gather data from patients, caregivers and healthcare professionals. Participants were selected from the patient and employee population of an academic medical center in The Netherlands. Nine patients and seven caregivers who dealt with lower limb amputation for no-option chronic limb threatening ischemia six to twelve months prior to the interview and were not cognitively impaired were selected. Nine healthcare professionals dealing with patients with no-option chronic limb threatening ischemia and lower limb amputation were selected.

Results: Lower limb amputation was explicitly discussed late in the disease process, sometimes during an emergency setting. Patients stated goals were never discussed, healthcare professionals stated they were. The most important goal for patients was to live independently after lower limb amputation. Patients and caregivers feel healthcare professionals should be upfront about the possible necessity of lower limb amputation. Reasons to undergo lower limb amputation were absence of treatment options, pain and wanting to enjoy life again. Participants indicated accelerating lower limb amputation was not a viable option.

Conclusion: All stakeholders reported overlapping values and preferences regarding main reasons for lower limb amputation, the primary goals after lower limb amputation, and the absence of a desire to accelerate lower limb amputation. The main difference in values and preferences is the preferred timing of discussing lower limb amputation.

Keywords: vascular surgery, shared decision-making, SDM, communication

\section{Introduction}

Assumptions from patients towards healthcare professionals and vice versa strongly influence decisions on treatment and open communication about values, preferences and goals is insufficiently performed. ${ }^{1,2}$ At the same time, it is widely believed and accepted that exploring patient values and preferences (shared goal-setting) should be part of any shared decision-making (SDM) process. ${ }^{3}$ However, SDM is not performed consistently amongst vascular patients and healthcare professionals tend 
to insufficiently appreciate patient values and preferences regarding the outcome of care.,

Lower limb amputation (LLA) is the ultimo scenario and necessary in the short term for patients with chronic limb threatening ischemia (CLI) for whom no revascularization options remain (no-option CLI). CLI is caused by peripheral arterial disease (PAD), which can be caused by atherosclerosis and/or diabetes mellitus (DM). This results in markedly reduced blood supply to the extremities and is characterized by unbearable pain in the legs and feet (ischemic rest pain) and/or non-healing ulcers. Its tremendous impact is reflected in quality of life (QoL) ratings comparable to terminal cancer patients and worse than patients with chronic heart or kidney disease.,7 QoL markedly improves after LLA, a phenomenon also described as the "disability paradox"., 8 Nevertheless, LLA is associated with invalidity and a transformed body image and self-identity. ${ }^{10}$ Little is known about the patient related issues (eg values, preferences, expectations of life/treatment) that healthcare professionals and patients consider and discuss when offering LLA as a treatment option. One study investigated decision-making before LLA. ${ }^{11}$ This study revealed that healthcare professionals play a key role, as they are the first to present LLA as a treatment option to the patient. However, this is only done after a long process of patients experiencing pain and suffering. LLA is then experienced as the culmination of this process and the only viable option left. Shared goal setting in patients with no-option CLI has not been previously studied.

The present study focusses on investigating patients and healthcare professionals' values, preferences and priorities when making such a critical decision. The goal of this qualitative study is to describe the range of views present in the study population and not to assess how often certain views are present. Specifically, the study focusses on the preferred timing of surgery, the method of communication, what patients and caregivers (nursing laypersons, eg family members) find important in life and the reason why they eventually chose to undergo LLA.

\section{Methods}

Semi-structured interviews were used to discuss a set of topics. The interview topics are included in Appendix A. These topics were selected based on the experience of the investigators with this patient population and knowledge from prior research. Interviews were recorded using a voice recorder and transcribed verbatim. Patients, their primary caregivers, and healthcare professionals from the Leiden University Medical Center (LUMC) were interviewed. Interviews with patients and caregivers were conducted separately and took place at their home or at the LUMC, based on their preferences. Interviews with healthcare professionals were held at the LUMC. Patient selection was performed on the $1^{\text {st }}$ of October 2018 using the local hospital treatment codes for LLA. This search yielded patients who underwent LLA for any reason. Patients and healthcare professionals were screened based on the in- and exclusion criteria indicated in Table 1. If a patient wished to participate in the study, his or her primary caregiver was also asked to participate. All participants (patients, primary caregivers, and healthcare professionals) provided informed consent for participation and publication of anonymized responses. Caregivers were interviewed because it was believed they could reflect on the disease process of the patient from another viewpoint since they had a close relationship with the patient. Caregivers were asked to reflect on how they believed the patient had experienced the disease process and answer the interview questions with that in mind. Because the caregivers' answers are about the experience of the patient, the caregiver answers are presented

Table I In- and Exclusion Criteria

\begin{tabular}{|l|l|l|}
\hline & Inclusion & Exclusion \\
\hline Patients & $\begin{array}{l}\text { Age } \geq 18 \text { years old. } \\
\text { LLA } 6 \text { to } 12 \text { months prior to interview. }\end{array}$ & $\begin{array}{l}\text { Diagnosis of dementia or other reasons for not being able to provide } \\
\text { reliable information. }\end{array}$ \\
\hline Caregivers & $\begin{array}{l}\text { Age } \geq 18 \text { years old. } \\
\text { Having a close relationship with the } \\
\text { interviewed patient. }\end{array}$ & $\begin{array}{l}\text { Diagnosis of dementia or other reasons for not being able to provide } \\
\text { reliable information. }\end{array}$ \\
\hline $\begin{array}{l}\text { Healthcare } \\
\text { professionals }\end{array}$ & $\begin{array}{l}\text { Being responsible for the care of no-option } \\
\text { CLI patients. }\end{array}$ & None. \\
\hline
\end{tabular}


alongside the patient' answers. All patients and caregivers were interviewed, after which data saturation was achieved. Data saturation was defined as the moment that no new themes had risen during multiple interviews. ${ }^{12}$ All data gathered in this study was stored anonymously using identification codes only accessible by the lead researcher. The transcribed interviews were coded using open coding in ATLAS.ti (version 8). Patient and caregiver demographics were analyzed using SPSS (version 25). The interviews were conducted, transcribed, and coded by researcher J.A.N., a research intern trained in all these aspects. Researcher W.A.G. (Health Psychology, Leiden University) provided J.A.N. with feedback on the interviews. After coding, categories were identified from the data and relationships between categories were assessed to investigate which themes were recurrent amongst the study population.

This study was ethically approved by the Commission of Medical Ethics of the Leiden University Medical Center, reference number P18.188. This study was conducted in accordance with the Declaration of Helsinki.

\section{Results}

Initial subject identification provided 35 patients who underwent LLA for any reason. Ten were deceased and six patients underwent LLA for other reasons than chronic limb threatening ischemia. A total of seven patients were excluded because they were not able to provide reliable information due to medical reasons such as dementia or ischemic strokes. In total 12 patients were eligible for inclusion after which nine patients (75\%) provided informed consent for participation and publication of anonymized responses. Seven caregivers and seven healthcare professionals were willing to participate in the study as well.

Participant characteristics are described in Table 2. All patients, caregivers and healthcare professionals had the Dutch nationality. In the following sections the various views of stakeholders regarding the primary study topics will be presented. The primary topics of this study are the values and preferences of stakeholders with regards to the preferred timing of surgery, the method of communication, what patients and caregivers find important in life and the reason why they eventually chose to undergo LLA. In Appendix B quotes from the interviews can be found to support the statements in this section.

\section{Discussing Lower Limb Amputations and Preferences \\ Patients and Caregivers}

Patients and caregivers were asked when and how LLA was discussed with them and how they would have preferred it to happen. LLA was often discussed explicitly for the first time a couple of days before the LLA took place or after minor amputation had been unsuccessful. This was often in a so-called emergency setting, meaning a patient had to be amputated within a few days. Two patients were informed about a possible LLA several months in advance and were given the opportunity to determine the timing of the surgery.

Many patients and their caregivers have stated they felt it was important that LLA was discussed as soon as their physician suspected it could happen in the future. Furthermore, they expressed the desire to have a possible LLA mentioned as soon as possible without causing unnecessary fear and they preferred their physician to be upfront and open about the prognosis.

Patients did not recall healthcare professionals initiating a joint discussion on post-amputation goals. However, more than half of these patients attributed this task to medical staff in rehabilitation facilities. Other patients felt discussing goals with healthcare professionals was unnecessary.

The most important goal that all patients mentioned was regaining independence after LLA. Furthermore, being able to work and being able to perform hobbies were also high-priority goals.

\section{Healthcare Professionals}

In the interviews, healthcare professionals indicated they are hesitant to discuss LLA with CLI patients when limb salvage is still achievable. They have stated that only when these options failed, they discussed LLA explicitly with the patient. Discussing it at an earlier stage in general was not seen as a viable option by most healthcare professionals. Each healthcare professional had their own reasons for not discussing LLA sooner. They believed it would not be useful, it would lead to distrust when patients eventually do not have to be amputated and could unnecessarily frighten a patient.

Nearly all healthcare professionals have stated they always discuss goals and preferences to some extent and they often use these goals to give the patients a perspective on what is possible after LLA. The goals and preferences 
Table 2 Participant Demographics

\begin{tabular}{|c|c|c|c|c|c|}
\hline & & $\mathbf{N}$ & $\%$ & Mean & SD \\
\hline \multicolumn{6}{|l|}{ Patients } \\
\hline \multirow[t]{3}{*}{ Gender } & & & & & \\
\hline & Male & 8 & 88,9 & & \\
\hline & Female & 1 & $\mathrm{II}, \mathrm{I}$ & & \\
\hline \multirow[t]{4}{*}{ Living Situation } & & & & & \\
\hline & Independent & I & $\mathrm{II}, \mathrm{I}$ & & \\
\hline & Independent, with others (eg partner, children, etc) & 7 & 77,8 & & \\
\hline & Nursing home & I & $\mathrm{II}, \mathrm{I}$ & & \\
\hline Age at inclusion in years & & & & 67,9 & 16,1 \\
\hline Age at LLA in years & & & & 67,0 & 16,3 \\
\hline Time since LLA in months & & & & 10,1 & 3,0 \\
\hline \multirow[t]{3}{*}{ Diagnosis for LLA } & & & & & \\
\hline & Atherosclerosis & 6 & 66,7 & & \\
\hline & Diabetic foot & 3 & 33,3 & & \\
\hline \multirow[t]{3}{*}{ Diabetes } & & & & & \\
\hline & Yes & 8 & 88,9 & & \\
\hline & Previously DM-I & $\mathrm{I}$ & $\mathrm{II}, \mathrm{I}$ & & \\
\hline \multirow[t]{3}{*}{ Leg } & & & & & \\
\hline & Left & 6 & 66,7 & & \\
\hline & Right & 3 & 33,3 & & \\
\hline \multirow[t]{3}{*}{ Below-/above-knee amputa } & & & & & \\
\hline & Below-knee amputation & 8 & 88,9 & & \\
\hline & Above-knee amputation & $\mathrm{I}$ & $\mathrm{II}, \mathrm{I}$ & & \\
\hline \multicolumn{6}{|l|}{ Caregivers } \\
\hline \multirow[t]{2}{*}{ Gender } & & & & & \\
\hline & Female & 7 & 100 & & \\
\hline \multirow[t]{4}{*}{ Relationship with patient } & & & & & \\
\hline & Partner & 4 & 57,1 & & \\
\hline & Daughter & 2 & 26,6 & & \\
\hline & Parent & $\mathrm{I}$ & 14,3 & & \\
\hline \multirow[t]{3}{*}{ Living Situation } & & & & & \\
\hline & Independent & 2 & 28,6 & & \\
\hline & Independent, with others (eg partner, children, etc) & 5 & 71,4 & & \\
\hline Age at inclusion in years & & & & 59,6 & 5,3 \\
\hline \multicolumn{6}{|l|}{ Healthcare professionals } \\
\hline \multirow[t]{3}{*}{ Gender } & & & & & \\
\hline & Male & 3 & 42,9 & & \\
\hline & Female & 4 & 57,1 & & \\
\hline \multirow[t]{3}{*}{ Profession } & & & & & \\
\hline & Surgeon (Including one resident) & 4 & 57,1 & & \\
\hline & Nurse & 3 & 42,9 & & \\
\hline Age at inclusion in years & & & & 41,7 & II \\
\hline
\end{tabular}


most healthcare professionals hear from patients are regaining independence after LLA and wanting to enjoy life again.

\section{Primary Reason for Lower Limb Amputation \\ Patients and Caregivers}

Patients and caregivers were asked what the primary reason was for undergoing LLA. Nearly all patients and caregivers have stated the absence of other therapeutic options or wanting to continue with their lives as primary reasons for LLA. In addition, many patients and some caregivers cited the experienced pain as an important factor in their decision. Furthermore, a couple of patients have stated they wanted to prevent an above-knee amputation. More than half of the patients and a couple of caregivers expressed feeling relieved when they made the decision to amputate and being content that the problems were over.

\section{Healthcare Professionals}

All healthcare professionals said most patients undergo LLA when they realize there are no other options left or that they feel they cannot continue as it is. According to all healthcare professionals virtually all patients are admitted and amputated in an emergency setting. Furthermore, many healthcare professionals indicated they believe the period leading up to LLA functions as a process of acceptation for the patient.

\section{Performing Lower Limb Amputations Sooner}

\section{Patients and Caregivers}

Patients and caregivers were asked if, in hindsight, they would have liked to be amputated sooner. Almost all patients responded that they would not have wanted to be amputated earlier and caregivers generally have stated they do not believe the patients would have wanted that Most patients expressed they kept hoping the leg would heal and LLA would be prevented. One patient expressed the desire to postpone LLA as long as possible. One patient might have preferred the LLA to be performed sooner, because of a feeling that postponing surgery resulted in not being able to enjoy life because of the CLI problems.

\section{Healthcare Professionals}

Healthcare professionals were asked whether they believed it would be preferable to amputate patients with limb ischemia sooner. All healthcare professionals have stated in general this was not the case. Reasons cited by most healthcare professionals were that not all patients would be open to this idea and they feel that most patients only want to undergo LLA when all other options are exhausted. On top of that one healthcare professional said that there could be a risk of "over-amputation" when choosing to amputate all patients earlier. Most healthcare professionals did believe that it would be beneficial for patients to have LLA performed sooner. However, they also declared that predicting which patients will need LLA beforehand is very difficult. Some healthcare professionals believed that some patients only afterwards assert that they should have had LLA sooner.

\section{Discussion}

This study provides more insight in the values and preferences from patients with no-option CLI, their primary caregivers and healthcare professionals regarding LLA. Some values and preferences are recognized and concur between patients and caregivers and professionals. For instance, patients, caregivers, and healthcare professionals in general did not see the need to accelerate LLA. In addition, patients and healthcare professionals indicated that the absence of other treatment options, ischemic pain, and wanting to enjoy life again, were the most important reasons to undergo LLA. Furthermore, patients and caregivers claimed that regaining independence was the most important goal to them, and healthcare professionals recognized this. Interestingly, there were also explicit differences, mainly when it came to discussing LLA. Patients explicitly preferred their physician to be upfront whether LLA was imminent, and they wanted it to be discussed as soon as possible. Healthcare professionals however, preferred to postpone talking about LLA until a patient had to undergo a limb saving revascularization or when almost all other treatment options had failed. Furthermore, patients in general do not recall healthcare professionals discussing goals with them during the disease process, whereas healthcare professionals do claim to discuss goals. This could point to a need of physicians to be more explicit when discussing this topic.

Due to the known level of increase in quality of life after lower-limb amputation, ${ }^{8}$ the expectation was that patients would indicate they would have liked to be amputated sooner. However, this was not the case. Healthcare professionals also did not see a need for acceleration of LLA because they believed patients in general want to exhaust all other options and healthcare professionals want to prevent "overamputation". On the other hand, most healthcare professionals 
did believe that amputated patients would have benefitted from LLA earlier in the disease process.

While the presumed reasons for LLA by healthcare professionals and the actual reasons stated by patients were largely the same, the preferences for when to discuss LLA differed greatly. Healthcare professionals experienced barriers to discuss LLA at an early stage with a patient. These barriers hamper open communication. Reported barriers for early discussions were the presence of other treatment options, a feeling it could lead to distrust amongst patients if LLA turned out to be unnecessary, a fear of unnecessarily frightening patients and a feeling it would not be useful to discuss LLA earlier. These barriers seem to be partly based on assumptions from healthcare professionals about the feelings of patients and partly on a fear of a difficult conversation which might turn out to be unnecessary. However, avoiding explicit discussions about the possibility of LLA at an early stage causes patients to be confronted with the verdict of LLA in an emergency setting. This was underscored by some patients who conveyed that LLA was only discussed with them after being admitted in an emergency setting, while they would have preferred healthcare professionals to be upfront and honest about a possible LLA earlier on. Professionals do seem to hint at LLA as a possibility during consultations, but do not discuss it explicitly. Healthcare professionals stated that predicting who needs LLA and when is very difficult and this is one of the reasons, they choose to postpone the conversation. This at times could lead to a delay in discussing lower limb amputation until it is inevitable A possible solution to enable earlier discussion about lower limb amputations is conducting more research into prediction models for which patients eventually need LLA. Certain risk factors may help to predict which patients need to undergo LLA, ${ }^{13-15}$ but so far reasonably accurate predictions of LLA have not been successful. Another solution to enable earlier discussions about LLA, is to require healthcare professionals to initiate the discussion at a fixed moment in the disease course through guidelines and protocols. An appropriate moment to do so would be prior to revascularization interventions. At that moment it should be explicitly discussed that in the event revascularization fails, LLA is a possibility. LLA does not have to be discussed with every patient visiting the outpatient clinic for CLI. Many of these patients will never need a LLA. However, it is important that healthcare professionals explicitly discuss LLA if they believe it could be a possibility in the future.
Even though it is difficult to predict which patients eventually need to undergo amputation, it is important to discuss it an earlier stage since patients prefer their healthcare professionals to be upfront about the possibility of LLA. Furthermore, most healthcare professionals presumed that patients need to undergo the whole CLI disease process with all its treatments to eventually be able to accept the decision to amputate and therefore choose to postpone an explicit discussion about LLA. However, the patients all mentioned that it did come as a great shock to hear about it only in an emergency setting. Therefore, it seems vital that healthcare professionals are open and more explicit about the possibility of LLA in every case where it might become actual in the future.

Although patients were of the opinion that healthcare professionals did not discuss goals with them, it is remarkable that all healthcare professionals said that regaining independence was the most important goal for patients after LLA, just as the patients indicated that was the case for them. It is possible that healthcare professionals interpreted the question about whether life goals were discussed during the consultations somewhat differently than the patients themselves. Possibly the patients thought the question referred to whether physical goals for after LLA were discussed, as opposed to a question about what they value in life. When discussing LLA, patients do not feel discussing goals is the responsibility of healthcare professionals in a hospital, but the job of healthcare professionals in a rehabilitation center. Even though patients and caregivers expressed they did not feel it was necessary to discuss goals with healthcare professionals in the hospital, it remains important in the process of SDM. ${ }^{3}$

Despite the limitations of this study, the findings must be viewed in the context of larger issues in medicine. As stated previously, mutual assumptions from patients and healthcare professionals strongly influence medical decision-making. Examples of these assumptions are the beliefs of both patients and healthcare professionals that a consultation should focus on symptom relief, the beliefs of patients that healthcare professionals are not interested in their personal goals, the beliefs of healthcare professionals that patients are not interested in sharing their personal goals and the beliefs of healthcare professionals that all patients have similar goals. ${ }^{1}$ However, discrepancy exists between what patients find important and what healthcare professionals think patients find important. ${ }^{2}$ This is underscored by our finding that patients find it important that the possibility of LLA is discussed as 
soon as possible, whereas healthcare professionals fear this could unnecessarily scare patients. Moreover, in critical situations, personal goals and priorities of patients may change, which hampers a doctor's ability to make assumptions without consulting the patient. ${ }^{16}$ Especially in older patients, the doctor often ultimately decides. ${ }^{17}$ Further incorporating SDM in conversations with patients could alleviate these problems. SDM is largely based on inquiring after a patients' values and preferences. ${ }^{3}$ Decisions in vascular surgery particularly call for SDM since there are often multiple treatment options to choose from. ${ }^{4}$ Studies have shown that vascular surgical patients want to be involved in treatment decisions but often do not experience their preferred level of involvement. ${ }^{18}$ Furthermore, incorporating SDM increases the likelihood patients receive treatment consistent with their values and preferences, patient satisfaction, healthcare outcomes, disease knowledge and decreases anxiety and costs. ${ }^{5,18-23}$ Taking all this into consideration, it seems important for healthcare professionals to be trained in SDM in order to adequately discuss values and preferences with patients. Much is known about the prognosis, functional outcomes and quality of life of no-option CLI patients after LLA, ${ }^{8,24-28}$ but up until now very little was known about the values and preferences of no-option CLI patients, their caregivers and healthcare professionals. The values and preferences discussed in this study could aid healthcare professionals in further incorporating SDM in daily practice, provide a starting point for discussing LLA at an earlier stage and possibly lead to providing more targeted and personalized care and a better management of the disease process, based on the values and preferences of individual patients. A future study should focus on whether the reported values and preferences (regaining independence, being able to work again and perform hobbies) are also applicable for patients who have yet to undergo LLA. A future study should also take prosthesis use of patients after LLA and their Rutherford classification prior to LLA into consideration and assess if values and preferences differ between patients with and without adequate prosthesis usage and the various Rutherford classes.

An important strength of this study is that it is one of the first, to our knowledge, to use a qualitative design to investigate values and preferences of patients who have dealt with LLA for no-option CLI. This allows for a deeper understanding of these issues. Another strength is that this study also takes the views of caregivers and healthcare professionals into account allowing for a broad view of the perspective of all relevant stakeholders regarding this subject. There are however some limitations to this study. First, it was a retrospective interview study with patients and caregivers who were asked to reflect on a previous period of care. This could lead to recall-bias and information being forgotten. Second, interviews were conducted and coded by one person. Third, all patients, caregivers and healthcare professionals had the Dutch nationality, which makes the results not generalizable. Fourth, all patients and caregivers were included from the LUMC. The results might differ for patients in different hospitals or different regions of the country. Last, all patients had been affected by diabetes, even the patients who were reported to be amputated because of atherosclerosis. It is possible that patients who were amputated due to diabetes have a different disease experience than those who are amputated due to atherosclerosis and that this influenced their decision.

The small sample size is not considered to be a limitation to this study since data saturation was achieved. It is therefore unlikely new views will come to light when interviewing more stakeholders.

\section{Conclusion}

This study provided insight in values and preferences of chronic limb threatening ischemia patients, their caregivers, and their healthcare professionals with regards to LLA. The goal of this study was to describe the range of views present in all three groups. Despite the small sample size, data saturation was achieved and therefore the following conclusions can be drawn. Overlapping ideas, values and preferences between the three groups occurred with regard to: 1) the opinion that there is no need to accelerate LLA and 2) the reasons that patients have for undergoing LLA and the primary goals of patients after LLA, eg, those related to regaining independence. The main difference between the groups is that healthcare professionals are not keen on discussing LLA at an earlier stage of the disease, whereas that is the preference of the patients. Based on the overlap in values and preferences discussed in this study and the preference of patients with regards to discussing LLA earlier, there is room to start discussions about lower limb amputations at an earlier stage in the disease process. The values and preferences discussed in this study, such as an upfront and open discussion about LLA and regaining independence after LLA, could provide healthcare professionals with starting points for earlier discussions and help taking values and preferences into account when discussing treatment options with patients. This will lead to further incorporating shared decision making in clinical practice which results in numerous advantages for patients. 


\section{Ethical Approval}

This study was ethically approved by the Commission of Medical Ethics of the Leiden University Medical Center, reference number P18.188. This study was conducted in accordance with the Declaration of Helsinki. All participants (patients, primary caregivers, and healthcare professionals) provided informed consent for participation and publication of anonymized responses.

\section{Acknowledgments}

The authors would like to acknowledge all participants for their participation in this study.

\section{Disclosure}

The authors report no conflicts of interest in this work. This research did not receive any specific grant from funding agencies in the public, commercial, or not-forprofit sectors.

\section{References}

1. Schulman-Green DJ, Naik AD, Bradley EH, et al. Goal setting as a shared decision making strategy among clinicians and their older patients. Patient Educ Couns. 2006;63:145-151. doi:10.1016/j. pec.2005.09.010

2. Nagl M, Farin E. Congruence or discrepancy? Comparing patients' health valuations and physicians' treatment goals for rehabilitation for patients with chronic conditions. Int $J$ Rehabil Res. 2012;35:26-35. doi:10.1097/MRR.0b013e32834d4be3

3. Legare F, Witteman HO. Shared decision making: examining key elements and barriers to adoption into routine clinical practice. Health Aff (Millwood). 2013;32:276-284. doi:10.1377/ hlthaff.2012.1078

4. Santema TB, Stubenrouch FE, Koelemay MJ, et al. Shared decision making in vascular surgery: an exploratory study. Eur J Vasc Endovasc Surg. 2016;51:587-593. doi:10.1016/j.ejvs.2015.12.010

5. Mulley AG, Trimble C, Elwyn G. Stop the silent misdiagnosis: patients' preferences matter. BMJ. 2012;345:e6572. doi:10.1136/ bmj.e6572

6. Albers MC, Fratezi AC, De Luccia N. Assessment of quality of life of patients with severe ischemia as a result of infrainguinal arterial occlusive disease. J Vasc Surg. 1992;16:54-59. doi:10.1016/07415214(92)90417-7

7. Sprengers RW, Teraa M, Moll FL, et al. Quality of life in patients with no-option critical limb ischemia underlines the need for new effective treatment. J Vasc Surg. 2010;52:843-849. doi:10.1016/j. jvs.2010.04.057

8. Fortington LV, Dijkstra PU, Bosmans JC, et al. Change in health-related quality of life in the first 18 months after lower limb amputation: a prospective, longitudinal study. $J$ Rehabil Med. 2013;45:587-594. doi:10.2340/16501977-1146

9. Albrecht GL, Devlieger PJ. The disability paradox: high quality of life against all odds. Soc Sci Med. 1999;48:977-988. doi:10.1016/ S0277-9536(98)00411-0

10. Senra H, Oliveira RA, Leal I, et al. Beyond the body image: a qualitative study on how adults experience lower limb amputation. Clin Rehabil. 2011;26:180-191. doi:10.1177/0269215511410731
11. Rassin M, Tzevlin V, Malul E, et al. Retrospective study of emerging themes in the decision-making process of patients considering amputation. $J$ Vasc Nurs. 2012;30:54-60. doi:10.1016/j. jvn.2012.02.002

12. Francis JJ, Johnston M, Robertson C, et al. What is an adequate sample size? Operationalising data saturation for theory-based interview studies. Psychol Health. 2010;25:1229-1245. doi:10.1080/ 08870440903194015

13. Aziz Z, Lin WK, Nather A, et al. Predictive factors for lower extremity amputations in diabetic foot infections. Diabetic Foot Ankle. 2011;2.

14. Desormais I, Aboyans V, Bura A, et al. Anemia, an independent predictive factor for amputation and mortality in patients hospitalized for peripheral artery disease. Eur J Vasc Endovasc Surg. 2014;48:202-207. doi:10.1016/j.ejvs.2014.04.005

15. Nather A, Bee CS, Huak CY, et al. Epidemiology of diabetic foot problems and predictive factors for limb loss. $J$ Diabetes Complications. 2008;22:77-82. doi:10.1016/j.jdiacomp.2007.04.004

16. Bynum JP, Barre L, Reed C, et al. Participation of very old adults in healthcare decisions. Med Decis Making. 2014;34:216-230. doi:10.1177/0272989X13508008

17. Wrede-Sach J, Voigt I, Diederichs-Egidi H, et al. Decision-making of older patients in context of the doctor-patient relationship: a typology ranging from "self-determined" to "doctor-trusting" patients. Int J Family Med. 2013;2013:478498. doi:10.1155/2013/478498

18. Santema TBK, Stoffer EA, Kunneman M, et al. What are the decision-making preferences of patients in vascular surgery? A mixed-methods study. BMJ Open. 2017;7.

19. Knops AM, Legemate DA, Goossens A, et al. Decision aids for patients facing a surgical treatment decision. Ann Surg. 2013;257:860-866. doi:10.1097/SLA.0b013e3182864fd6

20. Bekker HL, Hewison J, Thornton JG. Understanding why decision aids work: linking process with outcome. Patient Educ Couns. 2003;50:323-329. doi:10.1016/S0738-3991(03)00056-9

21. Lee EO, Emanuel EJ. Shared decision making to improve care and reduce costs. $N$ Engl $J$ Med. 2013;368:6-8. doi:10.1056/ NEJMp1209500

22. Naik AD, Kallen MA, Walder A, et al. Improving hypertension control in diabetes mellitus: the effects of collaborative and proactive health communication. Circulation. 2008;117:1361-1368. doi:10.1161/CIRCULATIONAHA.107.724005

23. Wilson SR, Strub P, Buist AS, et al. Shared treatment decision making improves adherence and outcomes in poorly controlled asthma. Am J Respir Crit Care Med. 2010;181:566-577. doi:10.1164/rccm.200906-0907OC

24. Klaphake S, de Leur K, Mulder PG, et al. Mortality after major amputation in elderly patients with critical limb ischemia. Clin Interv Aging. 2017;12:1985-1992. doi:10.2147/CIA.S137570

25. Taylor SM, Kalbaugh CA, Blackhurst DW, et al. Preoperative clinical factors predict postoperative functional outcomes after major lower limb amputation: an analysis of 553 consecutive patients. J Vasc Surg. 2005;42:227-235. doi:10.1016/j.jvs.2005.04.015

26. Schmiegelow MT, Sode N, Riis T, et al. Re-amputations and mortality after below-knee, through-knee and above-knee amputations. Dan Med J. 2018;65(12):65.

27. Madsen UR, Baath C, Berthelsen CB, et al. A prospective study of short-term functional outcome after dysvascular major lower limb amputation. Int J Orthop Trauma Nurs. 2018;28:22-29. doi:10.1016/ j.ijotn.2017.08.001

28. Chopra A, Azarbal AF, Jung E, et al. Ambulation and functional outcome after major lower extremity amputation. J Vasc Surg. 2018;67:1521-1529. doi:10.1016/j.jvs.2017.10.051 


\section{Publish your work in this journal}

Patient Preference and Adherence is an international, peer-reviewed, open access journal that focusing on the growing importance of patient preference and adherence throughout the therapeutic continuum. Patient satisfaction, acceptability, quality of life, compliance, persistence and their role in developing new therapeutic modalities and compounds to optimize clinical outcomes for existing disease

states are major areas of interest for the journal. This journal has been accepted for indexing on PubMed Central. The manuscript management system is completely online and includes a very quick and fair peer-review system, which is all easy to use. Visit http:// www.dovepress.com/testimonials.php to read real quotes from published authors. 